Supporting Information for:

Cucurbit[7]uril Mediates the Stereoselective [4+4] Photodimerization of 2-

Aminopyridine Hydrochloride in Aqueous Solution

Ruibing Wang, Lina Yuan, and Donal H. Macartney*

Department of Chemistry, Queen's University, Kingston, ON, Canada K7L 3N6; donal@chem.queensu.ca

Table of Contents

Page

Figure S1. ES-MS spectrum of a 2:1 mixture of 2-aminopyridine and $\mathrm{CB}[7]$ in acidic aqueous solution.

Figure S2. ${ }^{1} \mathrm{H}$ NMR chemical shift titration of 2-aminopyridine $(8 \mathrm{mM})$ with $\mathrm{CB}[7]$ in acidic $\mathrm{D}_{2} \mathrm{O}$.

Figure S3. Job's plot of the ${ }^{1} \mathrm{H}$ NMR chemical shift for the $\mathrm{H} 4$ proton of $\mathrm{APH}^{+}$ as a function of $[\mathrm{CB}[7]] /\left([\mathrm{CB}[7]]+\left[\mathrm{APH}^{+}\right]\right)$

Photodimerization experiments

Figure S4. ${ }^{1} \mathrm{H}$ NMR spectra $\left(400 \mathrm{MHz}\right.$ in $\left.\mathrm{D}_{2} \mathrm{O}\right)$ of $\mathrm{APH}^{+}$before (a) and after 3

hours (b), 6 hours (c), 9 hours (d), 15 hours (e), and 21 hours (f), of UV light irradiation.

Figure S5. ${ }^{1} \mathrm{H}$ NMR spectra $(400 \mathrm{MHz})$ of $\left.\mathrm{APH}^{+}(\mathrm{a}),\{(\mathrm{APH}))_{2} \cdot \mathrm{CB}[7]\right\}^{2+}$ before (b) $\mathrm{S} 6$ and after 3 hours (c), 6 hours (d), 9 hours (e), 15 hours (f) and 21 hours (g) UV light irradiation in $\mathrm{D}_{2} \mathrm{O}$.

Figure S6. 2D COSY NMR spectrum $(400 \mathrm{MHz})$ of \{anti-trans-DADAT $\cdot \mathrm{CB}[7]\}^{2+}$ in $\mathrm{D}_{2} \mathrm{O}$ (black triangles indicate the peaks of $\mathrm{CB}[7]$ ). $\quad \mathrm{S} 7$ Figure S7. ES-MS spectrum of the CB[7] inclusion complex of \{anti-trans $\quad$ S8 DADAT $\}^{2+}$ in acidic aqueous solution.

Figure S8. 2D COSY NMR spectrum $(400 \mathrm{MHz})$ of anti-trans-DADAT ${ }^{2+}$ (blue $\quad$ S9 number labeled) and syn-trans-DADAT ${ }^{2+}$ (red number labeled) in $\mathrm{D}_{2} \mathrm{O}$.

Stability constant for the anti-trans-DADAT•CB[7] guest-host complex:

Figure S9 Plot of $\Delta$ against $(\Delta / c)^{1 / 2}$ for $\{\text { anti-trans-DADAT } \cdot \mathrm{CB}[7]\}^{2+}$ guest-host $\quad \mathrm{S} 10$ complex using the $\mathrm{H} 5$ ' proton resonance.

Computational Studies

S11

Figure S10. A "ball and bond" picture of energy-minimized structure of \{anti-trans DADAT $\cdot \mathrm{CB}[7]\}^{2+}$ showing the Cartesian axes ( $\mathrm{z}$ is vertical and $\mathrm{y}$ point to the right) and some of the atom numbering.

Table S1. Coordinates of the atoms in energy-minimized structure of \{anti-trans $\mathrm{DADAT} \cdot \mathrm{CB}[7]\}^{2+}$ 


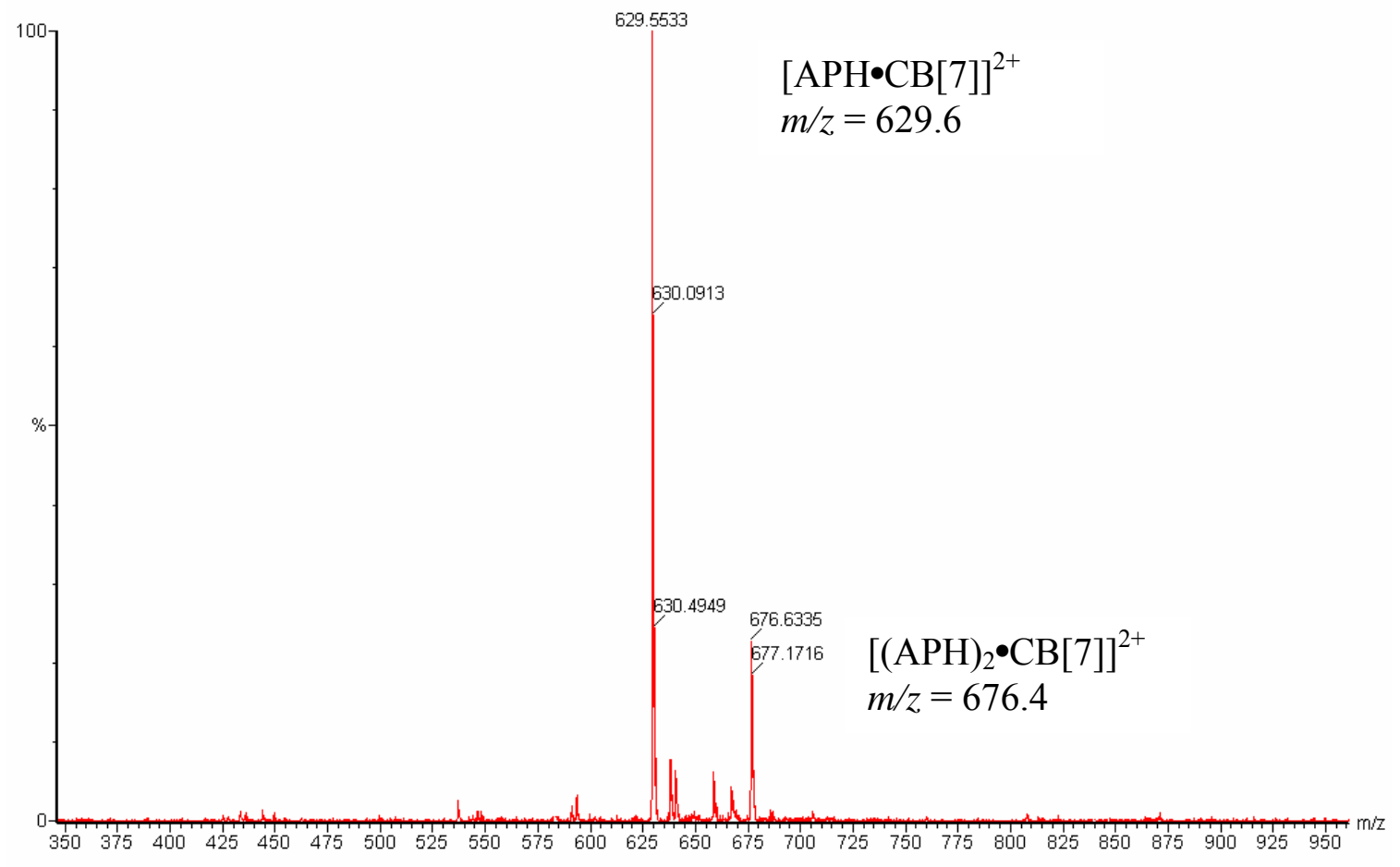

Figure S1. ES-MS spectrum of a 2:1 mixture of 2-aminopyridine and CB[7] in acidic aqueous solution. 


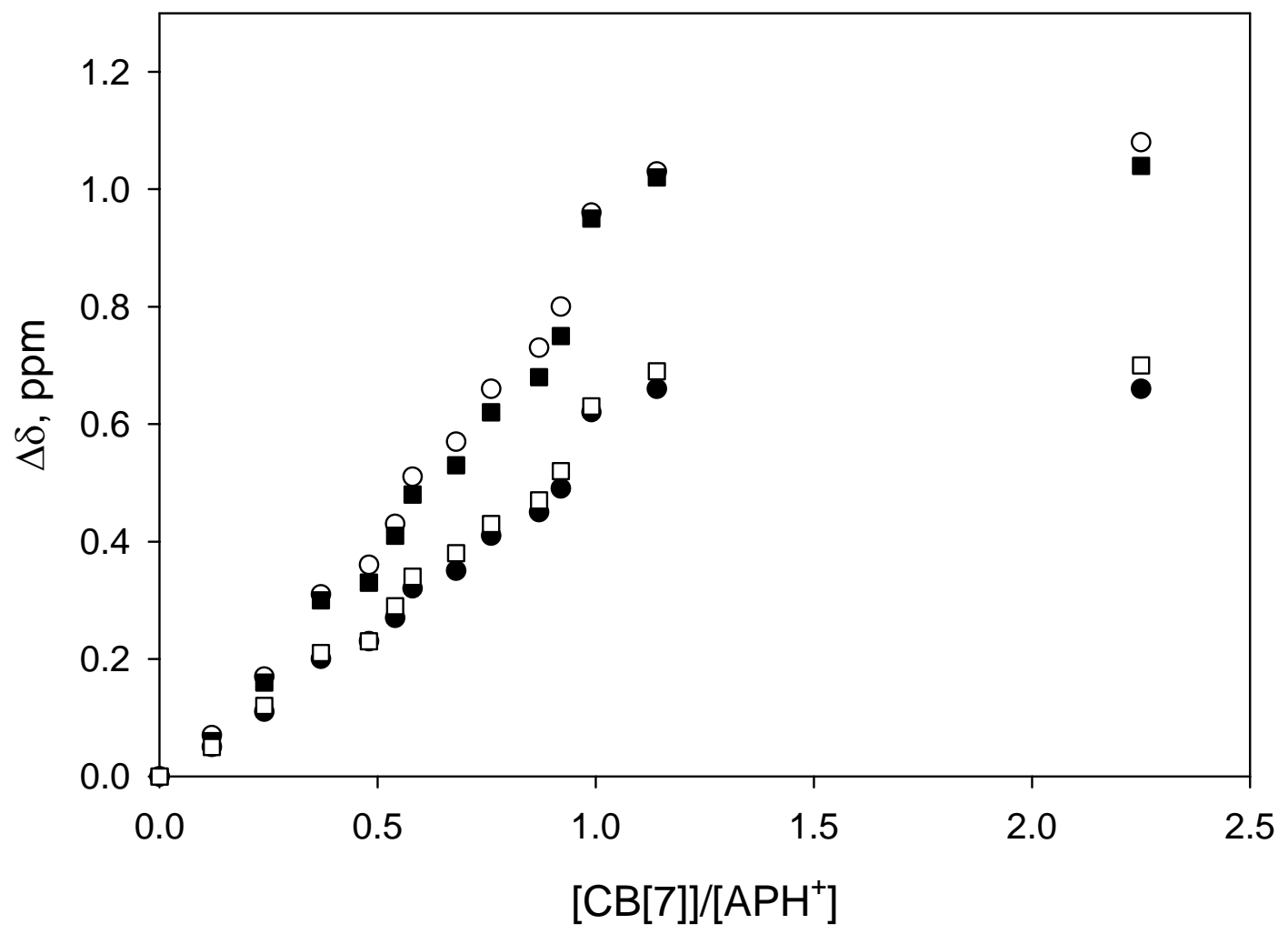

Figure S2. ${ }^{1} \mathrm{H}$ NMR chemical shift titration of 2-aminopyridine $(8 \mathrm{mM})$ with $\mathrm{CB}[7]$ in acidic $\mathrm{D}_{2} \mathrm{O}$. The proton resonances followed are the aromatic pyridine protons $\mathrm{H} 4(\bullet)$, H6 (०), H3 (ロ), and H5 (口). 


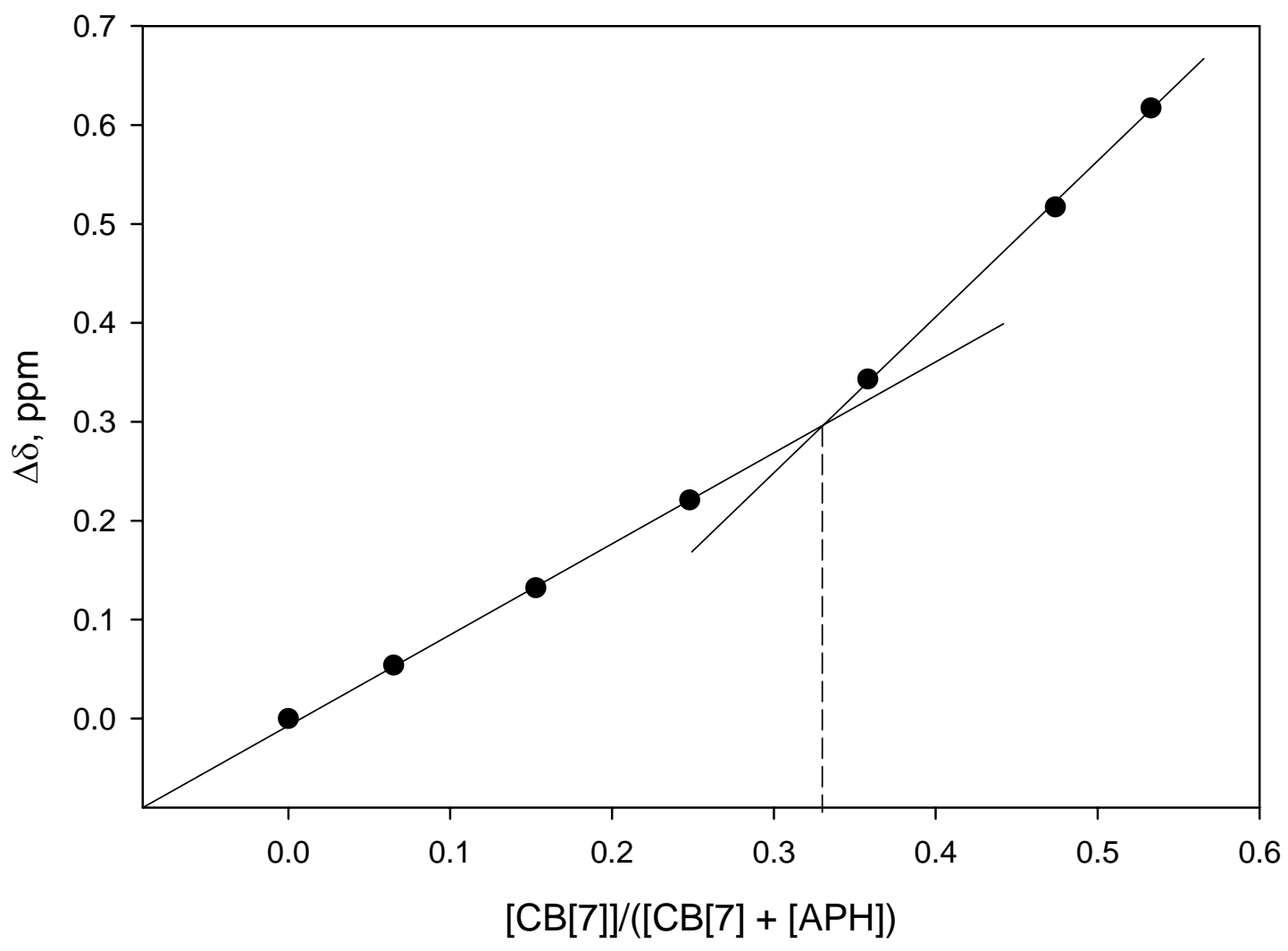

Figure S3. Job's Plot of the change in the ${ }^{1} \mathrm{H}$ NMR chemical shift for proton $\mathrm{H} 4$ of $\mathrm{APH}^{+}$as a function of $[\mathrm{CB}[7]] /\left([\mathrm{CB}[7]]+\left[\mathrm{APH}^{+}\right]\right)$with the total concentration $[\mathrm{CB}[7]]+$ $\left[\mathrm{APH}^{+}\right]=12.8 \mathrm{mM}$ in $\mathrm{D}_{2} \mathrm{O}$. 


\section{Photodimerization experiments:}

The NMR tubes with $\mathrm{APH}^{+}$and $\left\{(\mathrm{APH})_{2} \bullet \mathrm{CB}[7]\right\}^{+}$were irradiated with UV light $(365$ $\mathrm{nm})$ directly and were monitored by ${ }^{1} \mathrm{H}$ NMR spectroscopy every three hours.

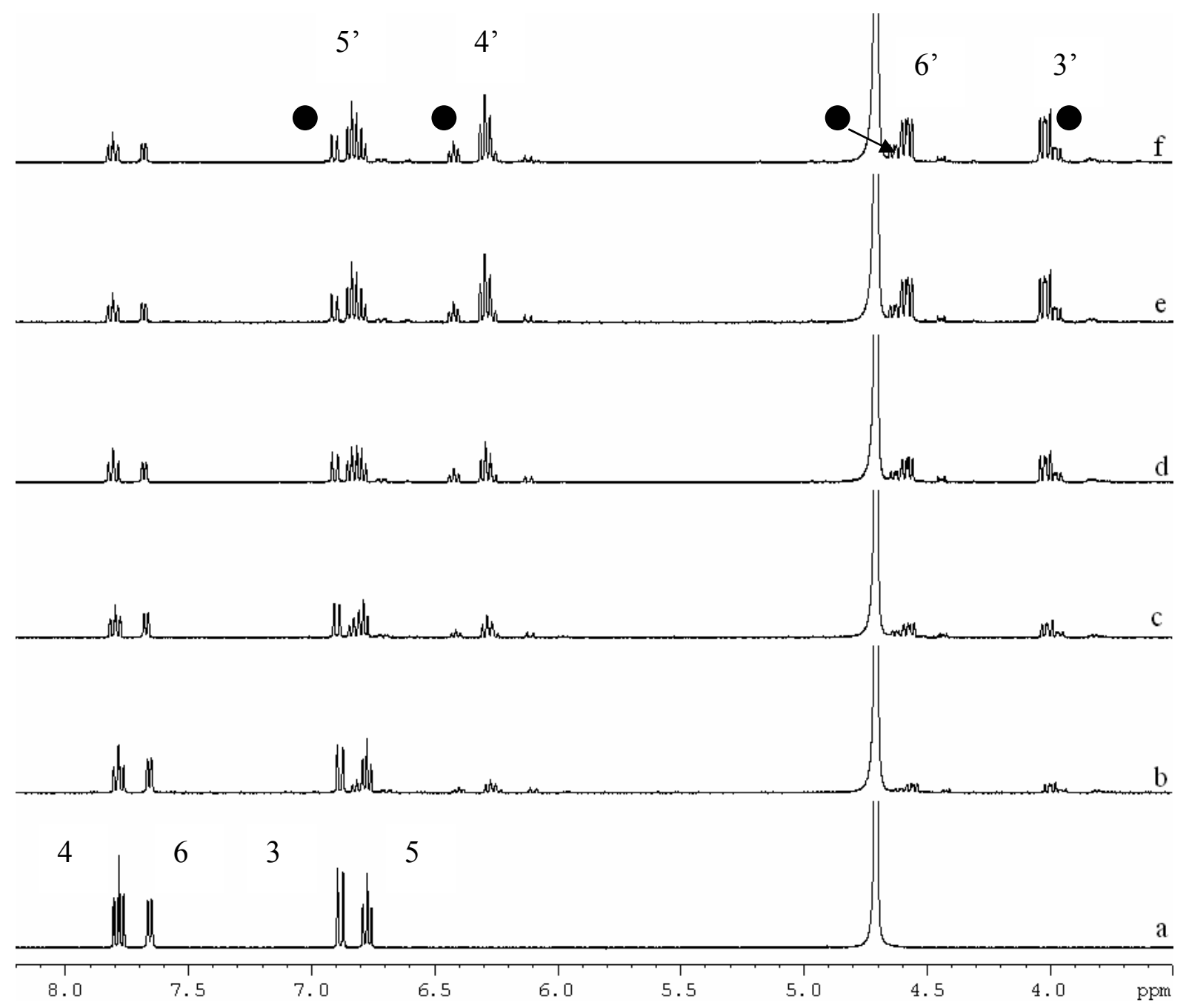

Figure S4. ${ }^{1} \mathrm{H}$ NMR spectra $\left(400 \mathrm{MHz}\right.$ in $\left.\mathrm{D}_{2} \mathrm{O}\right)$ of $\mathrm{APH}^{+}$before (a) and after 3 hours (b), 6 hours (c), 9 hours (d), 15 hours (e), and 21 hours (f), of UV light irradiation. The larger labeled peaks in (f) are for anti trans $\mathrm{DADAT}^{2+}$ product, while the smaller peaks $(\bullet)$ are for the syn trans DADAT ${ }^{2+}$ product. 


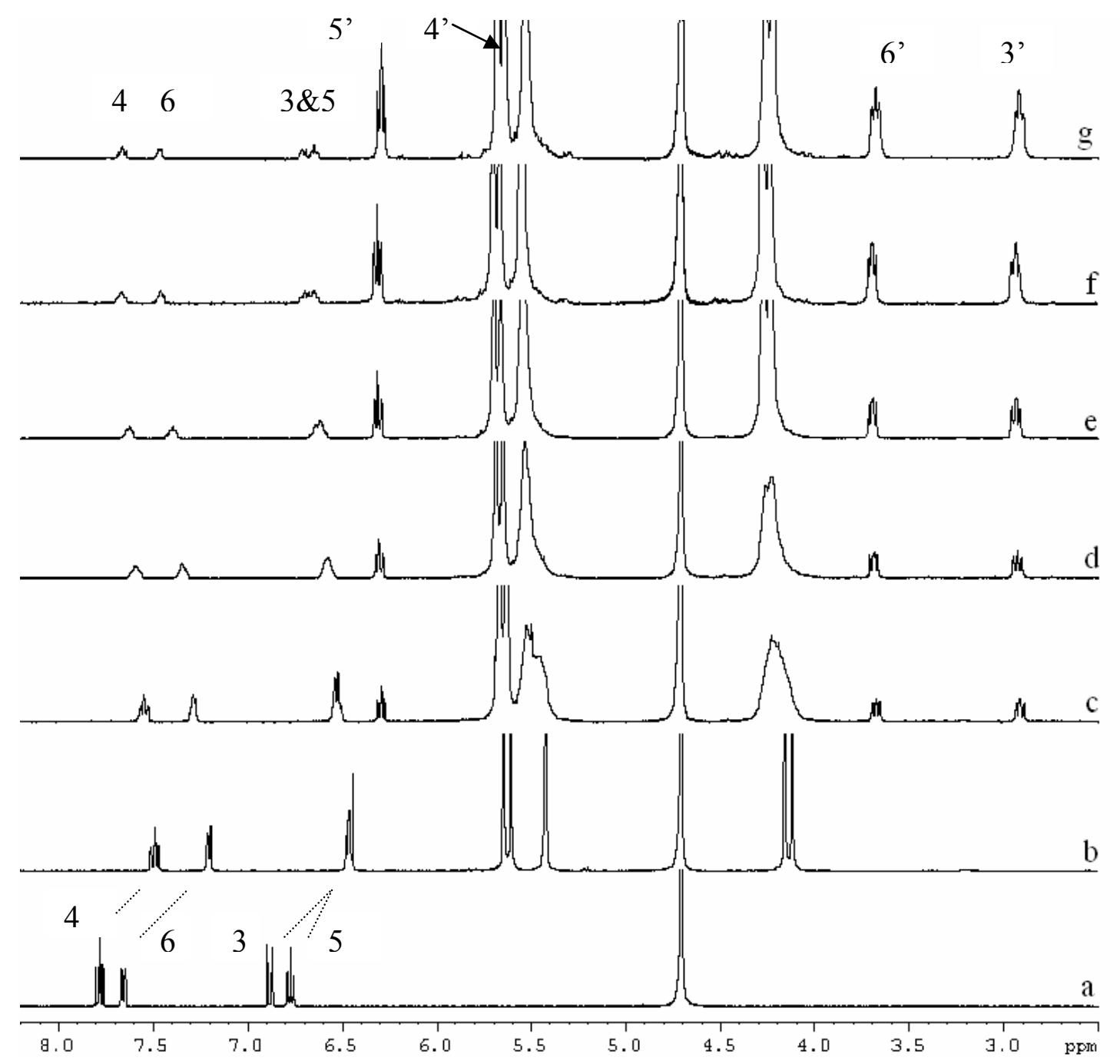

Figure S5. ${ }^{1} \mathrm{H}$ NMR spectra $(400 \mathrm{MHz})$ of $\mathrm{APH}^{+}(\mathrm{a}),\left\{(\mathrm{APH})_{2} \cdot \mathrm{CB}[7]\right\}^{2+}$ before (b) and after 3 hours (c), 6 hours (d), 9 hours (e), 15 hours (f) and 21 hours (g) UV light irradiation in $\mathrm{D}_{2} \mathrm{O}$. The proton resonances of $\mathrm{CB}[7]$ are shifted downfield during the process of dimerization, which means the $\mathrm{DADAT}^{2+}$ encapsulation by $\mathrm{CB}[7]$ may have altered the shape of the cavity slightly. It is also apparent that with the consumption of $\mathrm{APH}^{+}$and the formation of $\{\mathrm{DADAT} \cdot \mathrm{CB}[7]\}^{2+}$ during the photodimerization reaction, the equilibrium between 2:1 and 1:1 guest host complexes between $\mathrm{APH}^{+}$and $\mathrm{CB}$ [7] 
shifts towards the latter, as indicated by the downfield shifts of the proton resonances of unreacted $\mathrm{APH}^{+}$.

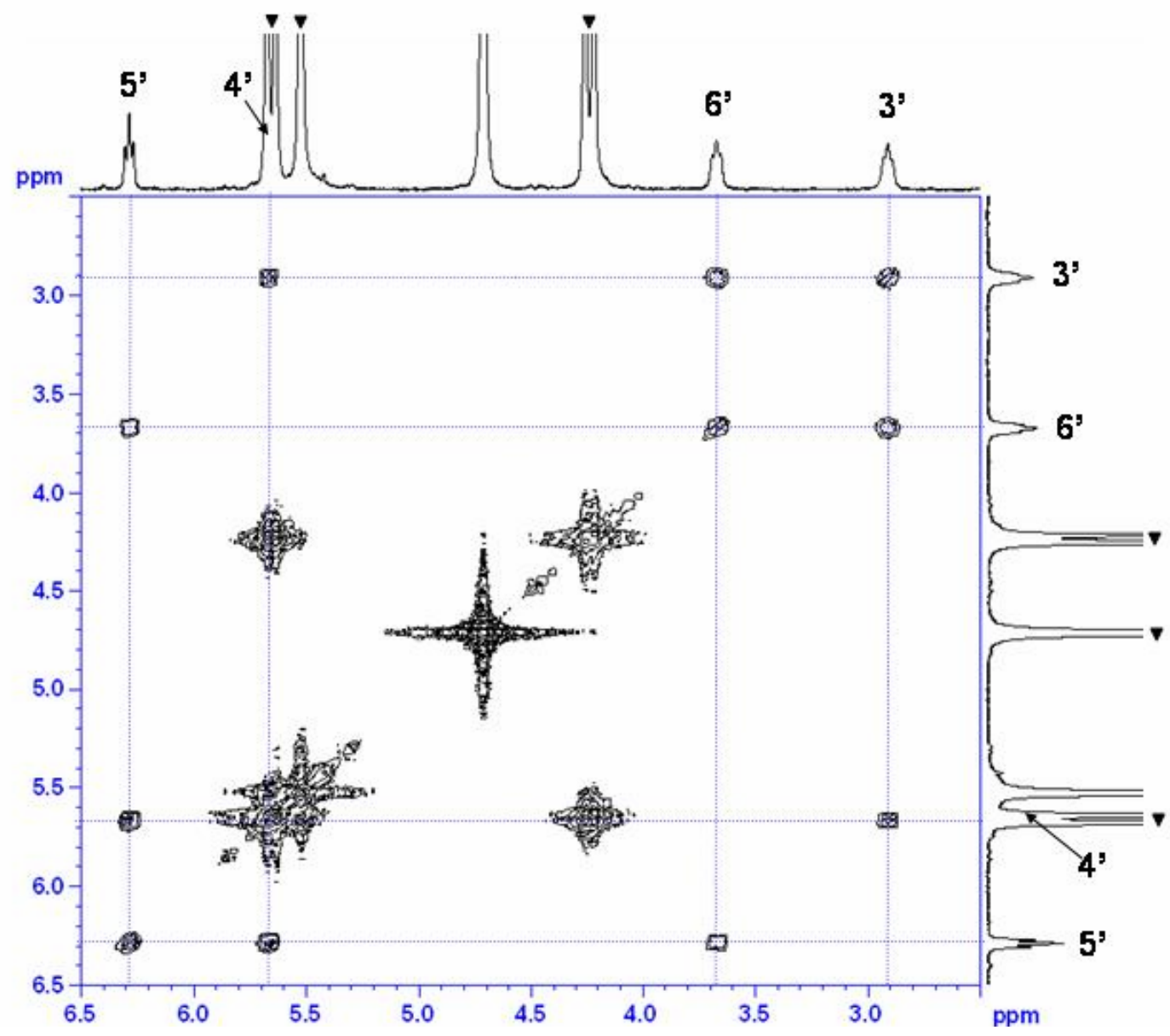

Figure S6. $2 \mathrm{D}$ COSY NMR spectrum $(400 \mathrm{MHz})$ of $\left\{\right.$ anti-trans-DADAT $\cdot \mathrm{CB}[7]^{2+}$ in $\mathrm{D}_{2} \mathrm{O}$ (black triangles indicate the peaks of $\left.\mathrm{CB}[7]\right)$. This spectrum was collected from the reaction solution after 34 hours irradiation. 


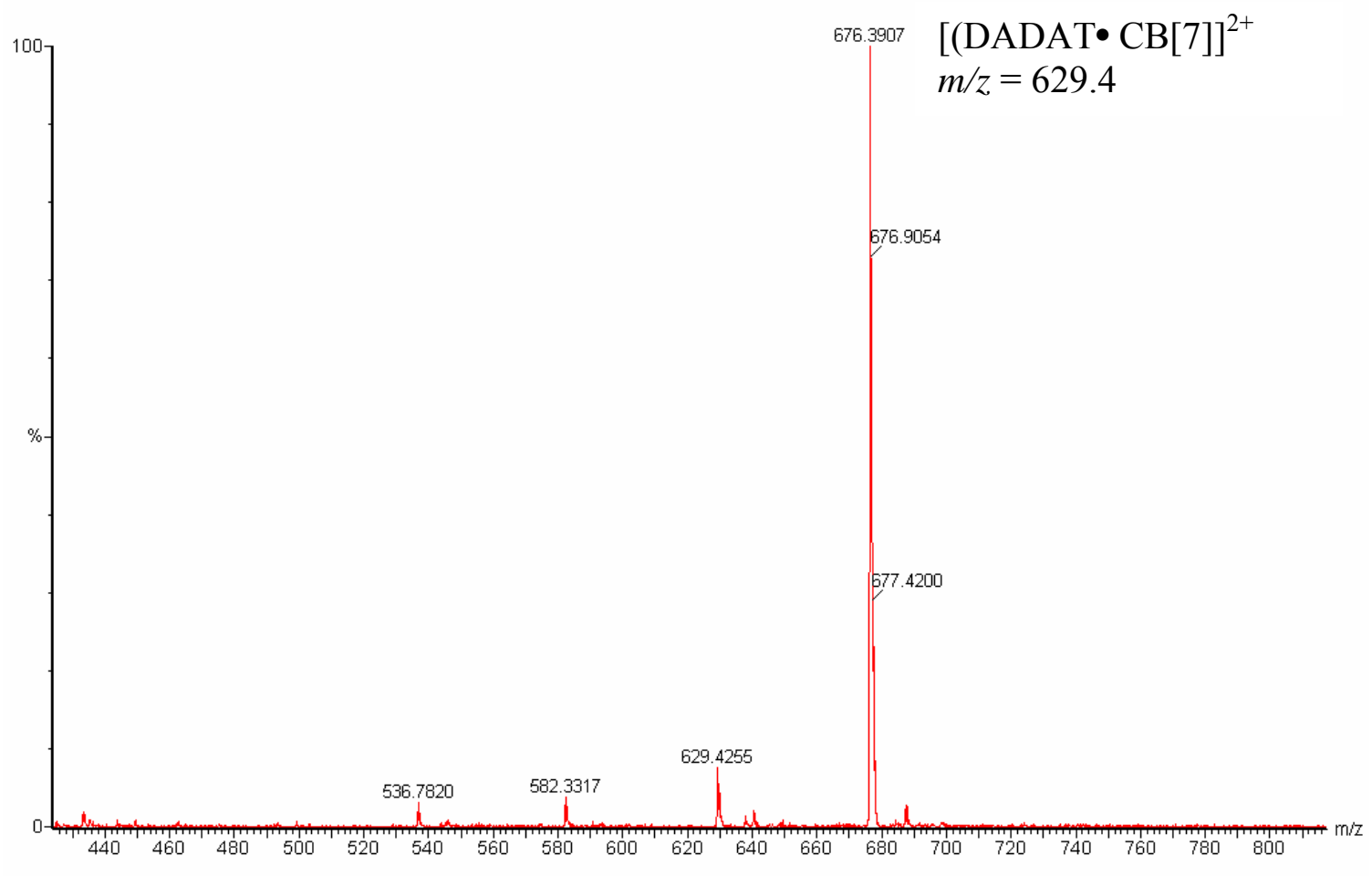

Figure S7. ES-MS spectrum of the CB[7] inclusion complex of anti-trans $\mathrm{DADAT}^{2+}$ in acidic aqueous solution. 


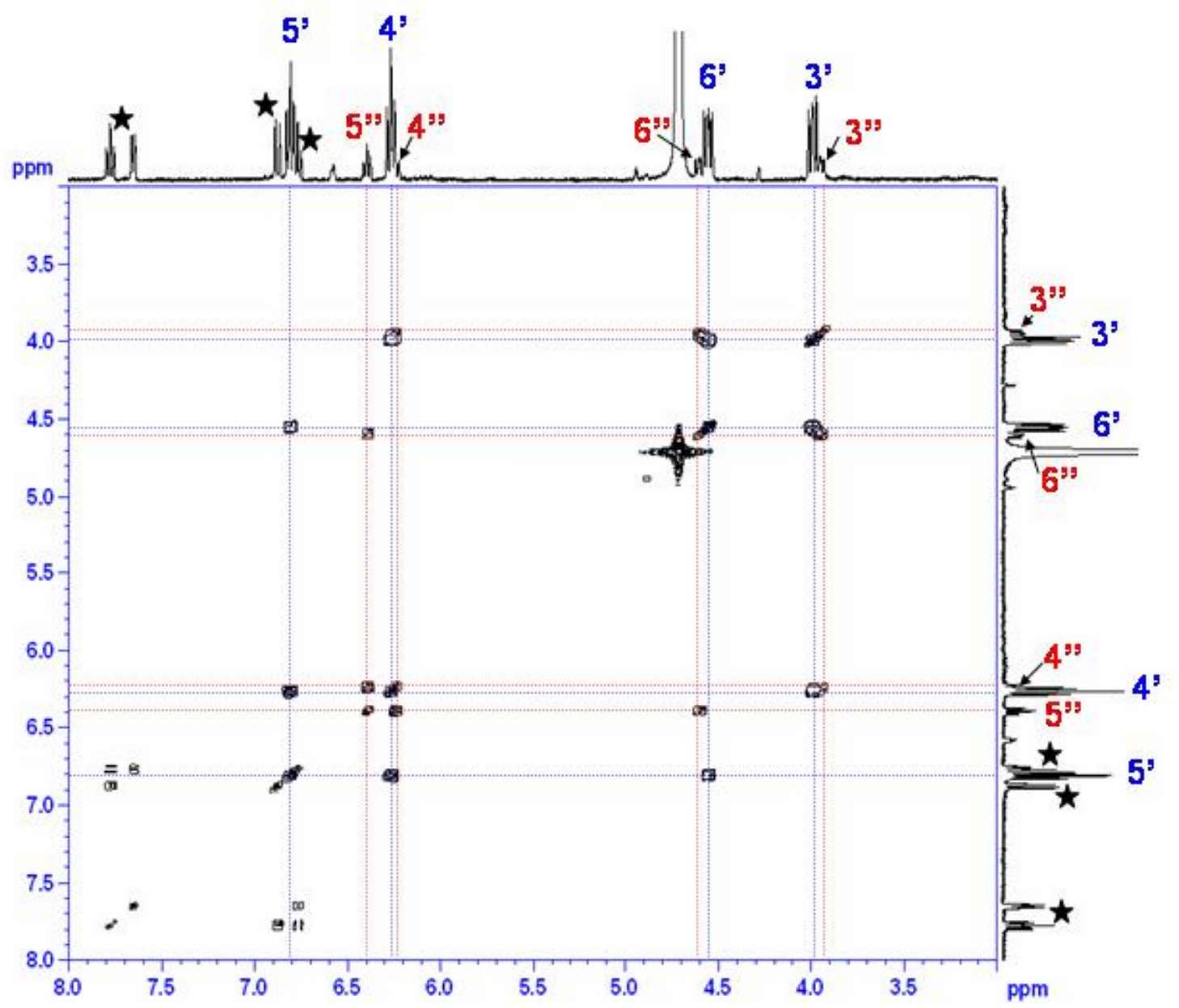

Figure S8. 2D COSY NMR spectrum $(400 \mathrm{MHz})$ of anti-trans-DADAT ${ }^{2+}$ (blue number labeled) and syn-trans-DADAT ${ }^{2+}$ (red number labeled) in $\mathrm{D}_{2} \mathrm{O}$. The black stars indicate the peaks of $\mathrm{APH}^{+}$(starting materials). The 3" and 6" protons have a direct correlation and this only occurs in anti-trans and syn-trans isomers of the four possible photodimer products. Since the anti-trans dimer was conclusively assigned above (Figure S6, in agreement with previous literature), this set of peaks is proposed to be syn-trans isomer of the dimer. This spectrum was collected for the reaction solution after 34 hours irradiation and some unidentifiable side-product peaks are also observed (in contrast to the photodimerization in the presence of $\mathrm{CB}[7])$. 


\section{Stability constant for the $\{\text { anti-trans-DADAT } \cdot \mathrm{CB}[7]\}^{2+}$ guest-host complex:}

The stability constant $(K)$ for the $\{\text { anti-trans-DADAT } \cdot \mathrm{CB}[7]\}^{2+}$ guest-host complex was determined from a ${ }^{1} \mathrm{H}$ NMR dilution experiment. ${ }^{2}$ A solution of \{anti-transDADAT $\cdot \mathrm{CB}[7]]^{2+}$ was prepared by forming a $1: 1$ mixture of anti-trans-DADAT ${ }^{2+}$ and $\mathrm{CB}$ [7] (concentration $(c)=5.8 \mathrm{mM}$ ) in a $\mathrm{D}_{2} \mathrm{O} / \mathrm{DCl}$. The ${ }^{1} \mathrm{H}$ NMR spectra of successive 2fold dilutions (down to $0.36 \mathrm{mM}$ ) of the solution with $\mathrm{D}_{2} \mathrm{O} / \mathrm{DCl}$ were recorded. The change in the observed chemical shift $(\Delta)$ with the change in the concentration (c) for a 1:1 guest-host complex is given by $\Delta=\Delta_{0}-(\Delta / c)^{1 / 2}\left(\Delta_{0} / K\right)^{1 / 2}$, where $\Delta_{0}$ is the limiting chemical shift difference between the resonance in the free guest and the corresponding resonance in the fully-formed 1:1 guest-host complex. The H5' resonance of anti-trans$\mathrm{DADAT}^{2+}$ was used in the determination of $K$ from a plot of $\mathrm{D}$ against $(\mathrm{D} / \mathrm{c})^{1 / 2}$. A value of $(8 \pm 2) \times 10^{5} \mathrm{M}^{-1}$ was calculated for $K$ for the $\{\text { anti-trans-DADAT } \bullet \mathrm{CB}[7]\}^{2+}$ complex.

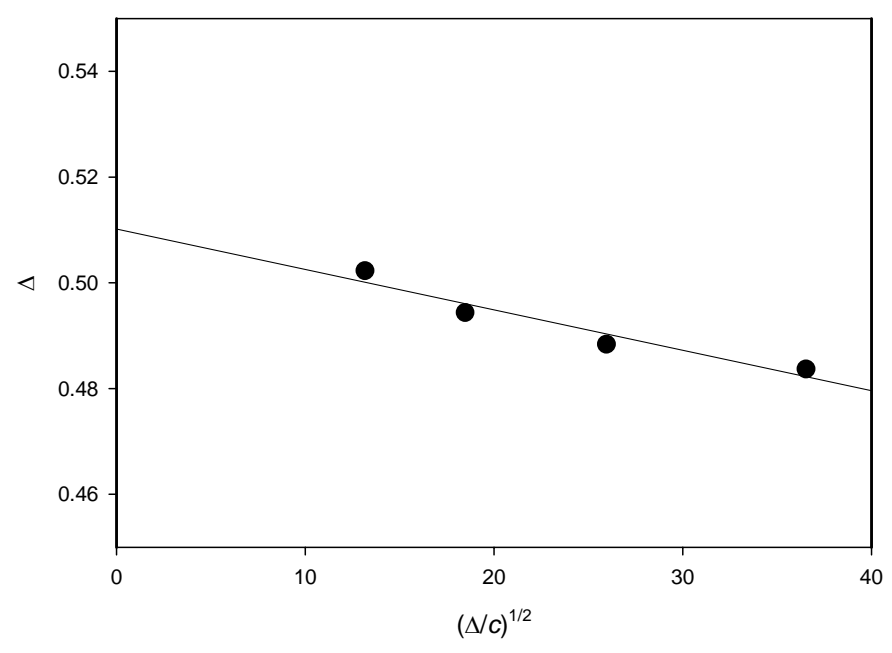

Figure S9. Plot of $\Delta$ against $(\Delta / c)^{1 / 2}$ for $\{\text { anti-trans-DADAT } \cdot \mathrm{CB}[7]\}^{2+}$ guest-host complex using the $\mathrm{H} 5$ ' proton resonance. 


\section{Computational Studies:}

The structure of the $\{\text { anti-trans DADAT } \cdot \mathrm{CB}[7]\}^{2+}$ guest-host complex was computed by energy minimizations using Gaussian 03, Revision C.02 programs ${ }^{3}$ run on the computing facilities of the High Performance Virtual Computing Laboratory (HPVCL) at Queen's University. The structure of the complex was originally constructed using ChemDraw and Chem 3D (ChemOffice 7.0, CambridgeSoft) programs and imported into Gaussian 03. The basis set used for the calculations was $H F / 3-21 G^{* *}$. The structure was minimized to final energy of -4765.99660448 A.U.

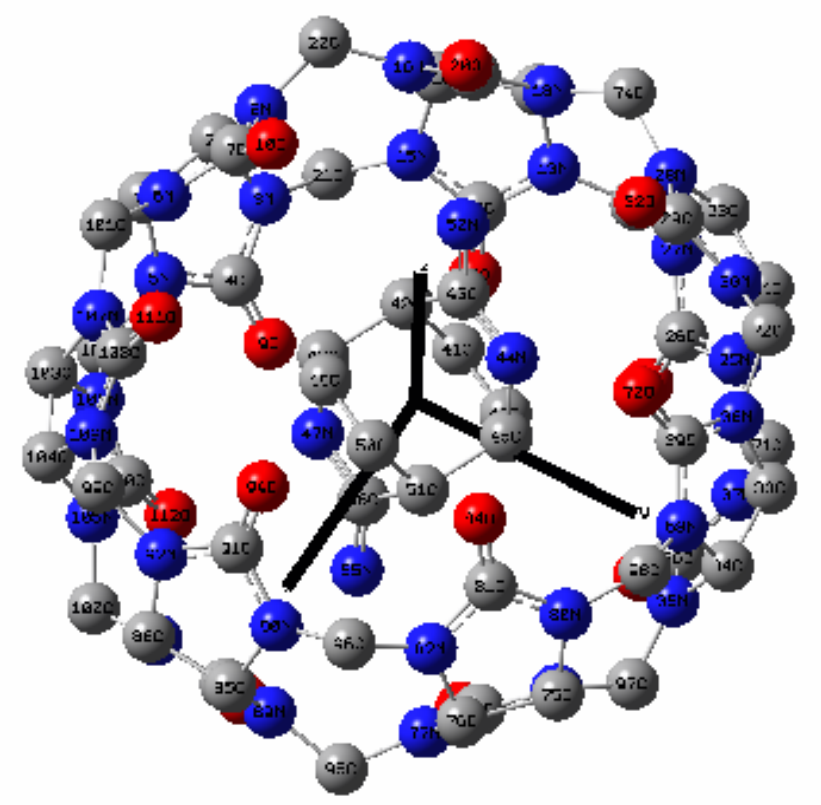

Figure S10. A "ball and bond" picture of energy-minimized structure of \{anti-trans $\mathrm{DADAT} \cdot \mathrm{CB}[7]\}^{2+}$ showing the Cartesian axes (z is vertical and y point to the right) and some of the atom numbering. 
Table S1. Coordinates of the atoms in energy-minimized structure of $\{\text { anti-trans DADAT } \cdot \mathrm{CB}[7]\}^{2+}$

\begin{tabular}{|c|c|c|c|c|c|}
\hline \multirow{2}{*}{$\begin{array}{l}\text { Center } \\
\text { Number }\end{array}$} & \multirow{2}{*}{$\begin{array}{l}\text { Atomic } \\
\text { Number }\end{array}$} & \multirow{2}{*}{$\begin{array}{l}\text { Atomic } \\
\text { Type }\end{array}$} & \multicolumn{3}{|c|}{ Coordinates (Angstroms) } \\
\hline & & & 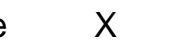 & Y & Z \\
\hline 1 & 6 & $\mathrm{C}$ & -1.086013 & -5.584168 & -0.124178 \\
\hline 2 & 6 & $\mathrm{C}$ & -2.614085 & -5.170176 & -0.179956 \\
\hline 3 & 7 & $\mathrm{~N}$ & -2.662725 & $-4.288628-$ & -1.329847 \\
\hline 4 & 6 & $\mathrm{C}$ & -1.429116 & -4.074436 & -1.890663 \\
\hline 5 & 7 & $\mathrm{~N}$ & -0.545338 & -4.948636 & -1.302694 \\
\hline 6 & 7 & $\mathrm{~N}$ & -0.635049 & -4.976981 & 1.115710 \\
\hline 7 & 6 & $\mathrm{C}$ & -1.627061 & -4.259060 & 1.751140 \\
\hline 8 & 7 & $\mathrm{~N}$ & -2.806241 & -4.488391 & 1.080293 \\
\hline 9 & 8 & $\mathrm{O}$ & -1.151839 & -3.278923 & -2.765099 \\
\hline 10 & 8 & $\mathrm{O}$ & -1.492008 & -3.558434 & 2.732759 \\
\hline 11 & 6 & C & -5.385591 & -2.754028 & -0.161253 \\
\hline 12 & 6 & C & -5.967843 & -1.278624 & -0.088669 \\
\hline 13 & 7 & $\mathrm{~N}$ & -5.467013 & -0.676303 & -1.300317 \\
\hline 14 & 6 & C & -4.611766 & -1.492100 & -1.992518 \\
\hline 15 & 7 & $\mathrm{~N}$ & -4.543383 & -2.698362 & -1.334930 \\
\hline 16 & 7 & $\mathrm{~N}$ & -4.680031 & -2.883642 & 1.096472 \\
\hline 17 & 6 & C & -4.700150 & -1.734311 & 1.841240 \\
\hline 18 & 7 & $\mathrm{~N}$ & -5.395451 & -0.779666 & 1.153296 \\
\hline 19 & 8 & $\mathrm{O}$ & -4.041153 & -1.224038 & -3.027228 \\
\hline 20 & 8 & 0 & -4.210632 & -1.608968 & 2.949699 \\
\hline 21 & 6 & $C$ & -3.888038 & -3.827950 & -1.966207 \\
\hline 22 & 6 & $C$ & -4.100085 & -4.107051 & 1.629137 \\
\hline 23 & 6 & $C$ & -5.511083 & 2.370352 & 0.007432 \\
\hline 24 & 6 & $C$ & -4.630724 & 3.685198 & 0.013593 \\
\hline 25 & 7 & $N$ & -3.875399 & 3.572171 & -1.213196 \\
\hline 26 & 6 & $C$ & -4.173272 & 2.431240 & -1.929283 \\
\hline 27 & 7 & $\mathrm{~N}$ & -5.167906 & 1.762191 & -1.252145 \\
\hline 28 & 7 & $N$ & -5.041286 & 1.663521 & 1.187874 \\
\hline 29 & 6 & $\mathrm{O}$ & -4.054972 & 2.340742 & 1.858792 \\
\hline 30 & 7 & $N$ & -3.856307 & 3.539673 & 1.226045 \\
\hline 31 & 8 & 0 & -3.695510 & 2.120293 & -2.994701 \\
\hline 32 & 8 & $\mathrm{O}$ & -3.496865 & 1.978891 & 2.878660 \\
\hline 33 & 6 & $C$ & -1.506569 & 5.575561 & -0.014035 \\
\hline 34 & 6 & $C$ & 0.072580 & 5.725157 & -0.017731 \\
\hline 35 & 7 & $\mathrm{~N}$ & 0.459105 & 4.984189 & -1.203291 \\
\hline 36 & 6 & $C$ & -0.621704 & 4.466558 & -1.892188 \\
\hline 37 & 7 & $N$ & -1.764280 & 4.856030 & -1.239409 \\
\hline
\end{tabular}




$\begin{array}{rrrrrr}38 & 7 & \mathrm{~N} & -1.742952 & 4.808011 & 1.193961 \\ 39 & 6 & \mathrm{C} & -0.589507 & 4.454151 & 1.836374 \\ 40 & 6 & \mathrm{C} & -1.134534 & 0.996183 & -1.172748 \\ 41 & 6 & \mathrm{C} & -1.667223 & -0.187970 & -0.969684 \\ 42 & 6 & \mathrm{C} & -1.312529 & -0.934764 & 0.312378 \\ 43 & 6 & \mathrm{C} & -1.334236 & 0.050763 & 1.465296 \\ 44 & 7 & \mathrm{~N} & -0.831520 & 1.241649 & 1.225179 \\ 45 & 6 & \mathrm{C} & -0.218566 & 1.522470 & -0.082667 \\ 46 & 6 & \mathrm{C} & 1.288355 & -0.157060 & -1.341627 \\ 47 & 7 & \mathrm{~N} & 0.739649 & -1.327588 & -1.097475 \\ 48 & 6 & \mathrm{C} & 0.144120 & -1.597547 & 0.210761 \\ 49 & 6 & \mathrm{C} & 1.065663 & -1.047273 & 1.291916 \\ 50 & 6 & \mathrm{C} & 1.610334 & 0.129028 & 1.077187 \\ 51 & 6 & \mathrm{C} & 1.240687 & 0.858145 & -0.207200 \\ 52 & 7 & \mathrm{~N} & -1.832427 & -0.278939 & 2.617178 \\ 53 & 1 & \mathrm{H} & -2.213301 & -1.181147 & 2.802548 \\ 54 & 1 & \mathrm{H} & -2.043347 & 0.425326 & 3.289560 \\ 55 & 7 & \mathrm{~N} & 1.878585 & 0.092764 & -2.469323 \\ 56 & 1 & \mathrm{H} & 2.062419 & -0.652755 & -3.106442 \\ 57 & 1 & \mathrm{H} & 2.287186 & 0.980550 & -2.694265 \\ 58 & 1 & \mathrm{H} & -0.757068 & 1.924207 & 1.953553 \\ 59 & 1 & \mathrm{H} & 0.615324 & -1.979690 & -1.840726 \\ 60 & 1 & \mathrm{H} & 1.239145 & -1.620248 & 2.176475 \\ 61 & 1 & \mathrm{H} & 0.048112 & -2.660460 & 0.300007 \\ 62 & 1 & \mathrm{H} & -2.030835 & -1.710357 & 0.504377 \\ 63 & 1 & \mathrm{H} & -2.268595 & -0.663898 & -1.710854 \\ 64 & 1 & \mathrm{H} & 2.226124 & 0.612152 & 1.803094 \\ 65 & 1 & \mathrm{H} & 1.943840 & 1.640103 & -0.415116 \\ 66 & 1 & \mathrm{H} & -0.086459 & 2.582421 & -0.149817 \\ 67 & 1 & \mathrm{H} & -1.297321 & 1.578787 & -2.054235 \\ 68 & 7 & \mathrm{~N} & 0.457052 & 5.085864 & 1.221996 \\ 69 & 8 & \mathrm{O} & -0.562112 & 3.814196 & -2.910948 \\ 70 & 8 & \mathrm{O} & -0.514578 & 3.712567 & 2.803104 \\ 71 & 6 & \mathrm{C} & -3.083614 & 4.631522 & -1.814946 \\ 83 & 8 & \mathrm{O} & 2.860202 & 2.785346 & 2.944018 \\ 72 & 6 & \mathrm{C} & -3.041411 & 4.581610 & 1.813974 \\ 73 & 6 & \mathrm{C} & -5.845972 & 0.622158 & -1.836957 \\ 74 & 6 & \mathrm{C} & -5.703154 & 0.505036 & 1.767655 \\ 75 & 6 & \mathrm{C} & 3.525274 & 4.652371 & 0.040564 \\ 76 & 6 & \mathrm{C} & 4.736791 & 3.629681 & 0.089271 \\ 77 & 7 & \mathrm{~N} & 4.577304 & 2.887526 & -1.146108 \\ 78 & 6 & \mathrm{C} & 3.432600 & 3.210413 & -1.814218 \\ 79 & 7 & \mathrm{~N} & 2.826678 & 4.256638 & -1.172413 \\ 80 & 7 & \mathrm{~N} & 2.810099 & 4.358862 & 1.258164 \\ 82 & 6 & \mathrm{C} & 3.316209 & 3.270849 & 1.933723 \\ & 7 & \mathrm{O} & 4.458959 & 2.869375 & 1.286314 \\ 83 & \mathrm{C} & 6.104962 & 0.205139 & 0.128766\end{array}$




$\begin{array}{cccccc}86 & 6 & \mathrm{C} & 5.942793 & -1.371284 & 0.105708 \\ 87 & 7 & \mathrm{O} & 5.280299 & -1.598302 & -1.162098 \\ 88 & 6 & \mathrm{C} & 5.052966 & -0.450146 & -1.875627 \\ 89 & 7 & \mathrm{O} & 5.519594 & 0.608459 & -1.135505 \\ 90 & 7 & \mathrm{O} & 5.359829 & 0.582195 & 1.304659 \\ 91 & 6 & \mathrm{C} & 4.784380 & -0.487257 & 1.954054 \\ 92 & 7 & \mathrm{~N} & 5.148724 & -1.632126 & 1.285444 \\ 93 & 8 & \mathrm{O} & 4.578287 & -0.384013 & -2.990522 \\ 94 & 8 & \mathrm{O} & 4.128425 & -0.428958 & 2.968890 \\ 95 & 6 & \mathrm{C} & 5.553414 & 1.948013 & -1.691246 \\ 96 & 6 & \mathrm{C} & 5.358073 & 1.903340 & 1.900984 \\ 97 & 6 & \mathrm{C} & 1.775338 & 5.033780 & -1.822675 \\ 98 & 6 & \mathrm{C} & 1.768028 & 5.177797 & 1.845322 \\ 99 & 6 & \mathrm{C} & 4.897863 & -2.940412 & 1.867426 \\ 100 & 6 & \mathrm{C} & 4.995491 & -2.897405 & -1.733268 \\ 101 & 6 & \mathrm{C} & 0.628955 & -5.304329 & 1.772210 \\ 102 & 6 & \mathrm{C} & 0.744230 & -5.227698 & -1.906508 \\ 103 & 6 & \mathrm{C} & 2.482698 & -5.165487 & -0.028562 \\ 104 & 6 & \mathrm{C} & 3.879810 & -4.419622 & 0.024169 \\ 105 & 7 & \mathrm{~N} & 3.847611 & -3.596399 & -1.170202 \\ 106 & 7 & \mathrm{~N} & 1.896002 & -4.646155 & -1.242671 \\ 107 & 7 & \mathrm{~N} & 1.833158 & -4.726585 & 1.190475 \\ 108 & 6 & \mathrm{C} & 2.621784 & -3.877347 & 1.938503 \\ 109 & 7 & \mathrm{~N} & 3.807352 & -3.699643 & 1.274715 \\ 110 & 6 & \mathrm{C} & 2.659252 & -3.672801 & -1.839786 \\ 111 & 8 & \mathrm{O} & 2.324608 & -3.416982 & 3.020482 \\ 112 & 8 & \mathrm{O} & 2.337237 & -3.028038 & -2.823879 \\ 113 & 1 & \mathrm{H} & -0.914763 & -6.648113 & -0.129127 \\ 114 & 1 & \mathrm{H} & -3.294223 & -5.998601 & -0.288575 \\ 115 & 1 & \mathrm{H} & -6.144020 & -3.514702 & -0.257232 \\ 116 & 1 & \mathrm{H} & -7.044686 & -1.240026 & -0.052489 \\ 117 & 1 & \mathrm{H} & -4.577468 & -4.660276 & -2.007256 \\ 118 & 1 & \mathrm{H} & -3.629108 & -3.506688 & -2.961560 \\ 119 & 1 & \mathrm{H} & -3.964875 & -3.945867 & 2.685153 \\ 130 & 1 & \mathrm{H} & -4.789118 & -4.921481 & 1.460429 \\ 133 & 1 & \mathrm{H} & -6.771635 & 0.664403 & 1.719313 \\ 121 & 1 & \mathrm{H} & -6.571194 & 2.556413 & 0.075290 \\ 122 & 1 & \mathrm{H} & -5.208633 & 4.595776 & 0.034756 \\ 124 & 1 & \mathrm{H} & -2.037070 & 6.513437 & 0.010569 \\ 125 & 1 & \mathrm{H} & 0.417860 & 6.745629 & -0.058308 \\ 126 & 1 & \mathrm{H} & -3.637401 & 5.558587 & -1.756358 \\ 127 & 1 & \mathrm{H} & -2.929895 & 4.350620 & -2.843742 \\ 128 & 1 & \mathrm{H} & -3.592371 & 4.292167 & 2.833840 \\ 130 & 1 & \mathrm{H} & -5.586912 & 5.509849 & 1.783920 \\ 131 & 1 & \mathrm{H} & -6.912122 & 0.747319 & -2.882493 \\ 13 & \mathrm{H} & 3.825648 & 5.686831 & -0.004725\end{array}$




\begin{tabular}{|c|c|c|c|c|c|}
\hline 134 & 1 & $\mathrm{H}$ & 5.706033 & 4.098681 & 0.134317 \\
\hline 135 & 1 & $\mathrm{H}$ & 7.130236 & 0.533379 & 0.196244 \\
\hline 136 & 1 & $\mathrm{H}$ & 6.880332 & -1.902842 & 0.147100 \\
\hline 137 & 1 & $\mathrm{H}$ & 6.537109 & 2.369201 & -1.542502 \\
\hline 138 & 1 & $\mathrm{H}$ & 5.344748 & 1.849900 & -2.743294 \\
\hline 139 & 1 & $\mathrm{H}$ & 5.029961 & 1.783838 & 2.920256 \\
\hline 140 & 1 & $\mathrm{H}$ & 6.362924 & 2.301708 & 1.871177 \\
\hline 141 & 1 & $\mathrm{H}$ & 2.098234 & 6.065435 & -1.866888 \\
\hline 142 & 1 & $\mathrm{H}$ & 1.664132 & 4.633877 & -2.817095 \\
\hline 143 & 1 & $\mathrm{H}$ & 1.653636 & 4.845774 & 2.863751 \\
\hline 144 & 1 & $\mathrm{H}$ & 2.081350 & 6.212079 & 1.818562 \\
\hline 145 & 1 & $\mathrm{H}$ & 4.629606 & -2.777733 & 2.898128 \\
\hline 146 & 1 & $\mathrm{H}$ & 5.803455 & -3.527085 & 1.800948 \\
\hline 147 & 1 & $\mathrm{H}$ & 4.789789 & -2.734593 & -2.777853 \\
\hline 148 & 1 & $\mathrm{H}$ & 5.862341 & -3.532836 & -1.618243 \\
\hline 149 & 1 & $\mathrm{H}$ & 0.571103 & -4.926474 & 2.779811 \\
\hline 150 & 1 & $\mathrm{H}$ & 0.728640 & -6.381654 & 1.781763 \\
\hline 151 & 1 & $\mathrm{H}$ & 0.720640 & -4.820400 & -2.903284 \\
\hline 152 & 1 & $\mathrm{H}$ & 0.875107 & -6.300326 & -1.943433 \\
\hline 153 & 1 & $\mathrm{H}$ & 2.563910 & -6.240502 & -0.064556 \\
\hline 154 & 1 & $\mathrm{H}$ & 4.729508 & -5.082857 & 0.006160 \\
\hline & & & A.U. & & \\
\hline
\end{tabular}


References

1. Taylor, E. C.; Kan, R. O. J. Am. Chem. Soc., 1963, 85, 776-784.

2. Alston, D. R.; Ashton, P. R.; Lilley, T. H.; Stoddart, J. F.; Zarzycki, R.; Slawin, A. M. Z.; Williams, D. J. Carbohydrate Research, 1989, 192, 259-281.

3. Frisch, M. J.; Trucks, G. W.; Schlegel, H. B.; Scuseria, G. E.; Robb, M. A.;

Cheeseman, J. R.; Montgomery Jr., J. A.; Vreven, T.; Kudin, N.; Burant, J. C.; Millam, J. M.; Iyengar, S. S.; Tomasi, J.; Barone, V.; Mennucci, B.; Cossi, M.; Scalmani, G.; Rega, N.; Petersson, G. A.; Nakatsuji, H.; Hada, M.; Ehara, M.; Toyota, K.; Fukuda, R.; Hasegawa, J.; Ishida, M.; Nakajima, T.; Y. Honda, Y.; Kitao, O.; Nakai, H.; Klene, M.; Li, X.; Knox, J. E.; Hratchian, H. P.; Cross, J. B.; Adamo, C.; Jaramillo, J.; Gomperts, R.; Stratmann, R. E.; Yazyev, O.; Austin, A. J.; Cammi, R.; Pomelli, C.; Ochterski, J. W.; Ayala, P. Y.; Morokuma, K.; Voth, G. A.; Salvador, P.; Dannenberg, J. J.; Zakrzewski, V. G.; Dapprich, S.; Daniels, A. D.; Strain, M. C.; Farkas, O.; Malick, D. K.; Rabuck, A. D.; Raghavachari, K. Foresman, J. B.; Ortiz, J. V.; Cui, Q.; Baboul, A. G.; Clifford, S.; Cioslowski, J.; Stefanov, B. B.; Liu, G..; Liashenko, A.; Piskorz, P.; Komaromi, I.; Martin, R. L.; Fox, D. J.; Keith, T.; Al-Laham, M. A.; Peng, C. Y.; Nanayakkara, A.; Challacombe, M.; Gill, P. M. W.; Johnson, B.; Chen, W.; Wong, M. W.; Gonzalez, C.; Pople, J. A. Gaussian 03, Revision C.02, Gaussian, Inc., Wallingford, CT, 2004. 